\title{
Misspecification in term structure models of commodity prices: Implications for hedging price risk
}

\author{
H. Suenaga \\ School of Economics and Finance, Curtin University, GPO Box U1987, Perth, WA 6845, AUSTRALIA. \\ Email: hiroaki.suenaga@,cbs.curtin.edu.au
}

\begin{abstract}
Stochastic dynamics of commodity prices and valuation of derivative contracts have long been studied in the field of financial economics. In the literature a common approach is to specify a stochastic dynamics of the underlying assets and derive from the suggested model valuation formulas of various derivative contracts whose payoff depends on the realization of the underlying asset value. Recently, models with additional latent factors and more flexible stochastic process of each factor have been suggested. Although these complex models tend to fit better to the observed data, it is often understated that this modeling approach only approximates the true stochastic process of the underlying asset values. This approximation bias can be substantial in magnitude for a storable commodity with significant demand and/or supply seasonality, for which equilibrium path of spot and futures prices cannot be expressed in reduced form, as shown by the theory of storage.

This study examines conventional term-structure models of commodity prices. In particular, this study quantifies the approximation bias of these conventional models through comparing them with an alternative approach of modeling the variance of daily futures returns directly by flexible non-parametric functions so as to allow the model to replicate highly non-linear price dynamics of storable commodities. Empirical applications of the models reveal that, for all four commodities (gold, crude oil, natural gas, and corn) examined in this study, the volatility of daily futures prices is more complex than the pattern as implied by the dynamics stipulated in the conventional term-structure models. In particular, all four commodities exhibit a strong time-to-maturity effect as well as a significant seasonal pattern in both levels and compositions (among two factors and idiosyncratic errors) of volatility of daily futures returns. Conventional termstructure models draw incorrect portraits of volatility dynamics as well as incorrect correlation among concurrently traded contracts with different maturity dates, which lead these models to suggest hedging strategies that are considerably less effective than the strategy based on the model of futures returns.
\end{abstract}

Keywords: commodity prices, term-structure model, volatility, hedging 


\section{INTRODUCTION}

Recent increases in the level and volatility of primary commodity prices have created tremendous uncertainties for producers, consumers, and other traders of these commodities. Stochastic dynamics of commodity prices and valuation of derivative contracts have long been studied in the field of financial economics. In the literature, a common approach is to specify the stochastic dynamics of the underlying asset, usually the spot price of the commodity under investigation, and derive from the suggested model valuation formulas of various derivative contracts whose payoff depends on the value of the underlying asset realized at contract maturity date (see Hull, 2000). In a seminal study, Schwartz (1997) suggests a model in which the underlying spot price is specified as a linear combination of three stochastic components, representing long-term factor, convenience yield factor, and interest rate.

Recent advancements in this modelling approach have been attained through increasing the number of latent factors and/or stipulating flexible stochastic process for each factor. These flexible models generally fit better to the observed price data, yet it is often understated that these models only approximate true stochastic dynamics of commodity prices. In particular, as shown by the theory of storage (Williams and Wright, 1991), for a storable commodity with strong seasonality in demand and/or supply, the equilibrium path of spot and futures prices exhibit highly non-linear dynamics that cannot be expressed in reduced form. Stochastic processes of the underlying spot price stipulated in models of commodity price dynamics, even recently developed complex models, cannot induce a futures price formula that replicates key features of commodity futures prices implied by the theory of storage. Thus, no matter how flexible a model is specified, it is intrinsically subject to approximation bias. This bias may result in inaccurate pricing of derivative contracts, lead to hedging strategies that are suboptimal, and erroneously conclude that the existing market is inefficient.

This study examines the conventional term-structure models of commodity prices through comparing them with a model of daily futures returns. In this alternative model, I follow the same approach as Smith (2005) and Suenaga and Smith (2011) and specify factor loadings directly by flexible, non-parametric functions, rather than determining them by a small number of parameters characterizing the temporal dynamics of the underlying stochastic factors. These flexible functions allow the model to replicate highly non-linear price dynamics of commodities with significant storage costs and strong seasonality in demand and/or supply. I quantify the approximation bias in conventional term-structure models through comparing them with the model of daily futures returns. Empirical applications to four commodities (gold, crude oil, natural gas, and corn) illustrate that the conventional term-structure models are subject to misspecification bias of considerable size, which can lead to hedging strategies that are less effective than the strategy based on the model of futures returns.

\section{COMPARISON OF TERM-STRUCTURE MODELS AND MODELS OF FUTURES RETURNS}

\subsection{Term Structure Models of Commodity Prices}

As an example of term-structure model of commodity prices, Schwartz and Smith (2000) consider a twofactor model in which $\log$ of the spot price, $S_{t}$, is a linear combination of the long-run equilibrium price, $\xi_{t}$ and the short-term deviation from the long-run equilibrium price, $\chi_{t}{ }^{1}$

$$
\begin{aligned}
& \ln S_{t}=\xi_{t}+\chi_{t} \\
& d \xi_{t}=\mu_{\xi} d t+\sigma_{\xi} d z_{\xi} \\
& d \chi_{t}=-\kappa \chi_{t} d t+\sigma_{\chi} d z_{\chi} \\
& d z_{\xi} d z_{\chi}=\rho_{\chi \xi} d t
\end{aligned}
$$

In (1), the long-term (LT) and short-term (ST) factors follow a Geometric Brownian Motion (GBM) and mean-reverting (MR) process, respectively, and two increments to the standard Brownian motion are allowed to correlate through the fourth equation. MR process allows the log of spot price to revert to long-run mean for $\kappa>0$. As argued by Schwartz (1997), the process is appropriate in modeling commodity prices, for which demand and supply response force prices, if unusually high or low, to revert to the long-run equilibrium level.

\footnotetext{
${ }^{1}$ Schwartz and Smith (2000) show that the model defined in (1) is equivalent to the two-factor model of Schwartz (1997) in which two latent factors represent the spot price and convenience yield.
} 
Two-factor models similar to (1) have been applied to various commodities, such as metals and energy commodities. ${ }^{2}$ A notable modification on model (1) as considered in previous studies is to allow mean price to exhibit seasonal variation in the right-hand side of the first equation in (1) when applying the model to a commodity with substantial seasonality in demand or supply.

To derive the pricing formula of a financial contract, the stochastic process of the underlying spot price in (1) is transformed into a process that is martingale under the risk-neutral probability measure:

$$
\begin{aligned}
& d \chi_{t}=\left(-\kappa \chi_{t}-\lambda_{\chi}\right) d t+\sigma_{\chi} d z_{\chi}^{*} \\
& d \xi_{t}=\left(\mu_{\xi}-\lambda_{\xi}\right) d t+\sigma_{\xi} d z_{\xi}^{*} \\
& d z_{\chi}^{*} d z_{\xi}^{*}=\rho_{\chi_{\xi}} d t
\end{aligned}
$$

where $d z_{\chi}^{*}$ and $d z_{\xi}^{*}$ are increments of the standard Brownian motion under risk neutral probability measure and the two coefficients $\lambda_{\chi}$ and $\lambda_{\xi}$ are often interpreted as representing the market price of risk associated with each stochastic factor.

The price in period $t$ of the futures contract maturing in period $T$ is obtained as the period $t$ conditional expectation of period $T$ spot price. It is obtained as, for the spot price following the stochastic process (1),

(2) $\ln F(t, T)=\xi_{t}+e^{-\kappa \tau} \chi_{t}-\frac{\left(1-e^{-\kappa \tau}\right) \lambda_{\chi}}{\kappa}-\lambda_{\xi} \tau+\left(\mu_{\xi} \tau+\frac{\left(1-e^{-2 \kappa \tau}\right) \sigma_{\chi}^{2}}{4 \kappa}+\frac{\sigma_{\xi}^{2} \tau}{2}+\frac{\left(1-e^{-\kappa \tau}\right) \rho_{\chi \xi} \sigma_{\chi} \sigma_{\xi}}{\kappa}\right)$

The model parameters, $\Omega=\left\{\kappa, \sigma_{\chi}, \sigma_{\xi}, \lambda_{\chi}, \lambda_{\xi}, \rho_{\chi_{\xi}}\right\}$, are usually estimated with the futures price data. In many organized exchanges, multiple contracts with different maturity dates are traded per each commodity. To fit equation (2) into multiple prices observed on day $t$ with different time-to-maturity $(T)$, an error term, often called measurement error, is added to the right-hand side of (2), which makes the values of the stochastic factors $\chi$ and $\xi$ not identifiable. The model is put into a state-space form and the parameters are estimated usually by a filtering method,

$$
\ln F(t, T)=\xi_{t}+e^{-\kappa \tau} \chi_{t}-\frac{\left(1-e^{-\kappa \tau}\right) \lambda_{\chi}}{\kappa}-\lambda_{\xi} \tau+\left(\mu_{\xi} \tau+\frac{\left(1-e^{-2 \kappa \tau}\right) \sigma_{\chi}^{2}}{4 \kappa}+\frac{\sigma_{\xi}^{2} \tau}{2}+\frac{\left(1-e^{-\kappa \tau}\right) \rho_{\chi \xi} \sigma_{\chi} \sigma_{\xi}}{\kappa}\right)+u_{t, T}
$$

$$
\begin{aligned}
& \chi_{t}=e^{-\kappa} \chi_{t-1}+\omega_{1, t} \\
& \xi_{t}=\mu_{\xi}+\xi_{t-1}+\omega_{2, t}
\end{aligned}
$$

where $\mathrm{E}\left[u_{t, T}\right]=0$ and $\mathrm{V}\left[u_{t, T}\right]=\sigma_{T}^{2} \forall t$ and $T . \boldsymbol{\omega}_{t}=\left\{\omega_{1, t}, \omega_{2, t}\right\}^{\prime}$ is iid bivariate normal with $\mathrm{E}\left[\boldsymbol{\omega}_{t}\right]=0$ and $\mathrm{E}\left[\omega_{\imath} \omega_{l}^{\prime}\right]=\mathbf{H}$ where $\mathbf{H}$ is the symmetric matrix with $\sigma_{x}^{2}$ and $\sigma_{\xi}^{2}$ on the main diagonal and $\rho \sigma_{x} \sigma_{\xi}$, off diagonal.

\subsection{Models of Price Return (POTS model)}

Partially overlapping time-series (POTS) model, introduced by Smith (2005), is a latent factor model of daily futures price changes. In this model, daily return of a futures contract is decomposed into the common latent factors and an idiosyncratic term. The model, as applied to the NYMEX energy futures contracts in Suenaga and Smith (2011), is expressed in the following form,

$$
\Delta F_{t, m}=\theta_{1}(m, d) \varepsilon_{1, t}+\theta_{2}(m, d) \varepsilon_{2, t}+\theta_{3}(m, d) u_{t, m}
$$

where $\Delta F_{t, m}=F_{t, m}-F_{t-1, m}$ is the daily price change on trade date $t$ of futures contract that matures at $m, \varepsilon_{1, t}$ and $\mathcal{E}_{2, t}$ are the latent factors that affect all the contracts traded on $t, u_{t, m}$ is the idiosyncratic error that is specific to the contract maturing at $m, \theta_{1}(m, d)$ and $\theta_{2}(m, d)$ are the factor loadings determining the extent that the underlying shocks, $\varepsilon_{1, t}$ and $\varepsilon_{2, t}$, are reflected into the change in the price of future contract maturing in $m$, and $\theta_{3}(m, d)$ determines the standard deviation of the shock specific to the contract maturing in $m$.

The three terms, $\theta_{i}(m, d), i=1, \ldots 3$, are specified as functions of the contract delivery month $(m)$ and the time to delivery of the contract $(d=m-t)$,

\footnotetext{
${ }^{2}$ See Lautier (2005) for a comprehensive review on applications of term-structure models of commodity prices to various commodities.
} 


$$
\theta_{i}(m, d)=\exp \left[a_{i, m, 0}+a_{i, m, 1} d+\sum_{k=1}^{K}\left(a_{i, m, 2 k} \sin \left(\frac{2 \pi k d}{d_{\max }}\right)+a_{i, m, 2 k+1} \cos \left(\frac{2 \pi k d}{d_{\max }}\right)\right)\right]
$$

where $d_{\max }$ is the maximum days to maturity for which the model is estimated. For identification, the constraint is imposed as $a_{2, m, 0}=-\sum_{k=1}^{K} a_{2, m, 2 k+1}-a_{2, m, 1} d_{\max }-10$ so that $\theta_{2}\left(m, d_{\max }\right) \square 0$ for all $m$. The condition is equivalent to the one used in Schwartz and Smith (2000), which allows the two factors to be interpreted as representing the LT and ST factor, respectively.

\subsection{Model Comparison}

A major difference between the conventional term-structure models of commodity prices and the POTS model is that the former specifies the dynamics of price level whereas the latter specifies the dynamics of price return. By modeling price return rather than level, the POTS model does not specify seasonal and any other deterministic variation of the commodity price that result from demand/supply seasonality and other peculiarities of the underlying commodity. Thus, the model is free from bias in approximating such deterministic price variation.

To compare the two models in further details, take the first difference of the futures price formula in (2),

$$
\Delta \ln F_{t, m}=\frac{\rho^{s}(1-\rho)}{\kappa}\left(\lambda_{\chi}-\rho_{\chi \xi} \sigma_{\chi} \sigma_{\xi}\right)+\lambda_{\xi}-\frac{\sigma_{\chi}^{2} \rho^{2 s}\left(1-\rho^{2}\right)}{4 \kappa}-\frac{\sigma_{\xi}^{2}}{2}+\rho^{s} \omega_{1, t}+\omega_{2, t}+\Delta u_{t, T}
$$

where $s=m-t$ is the time to maturity of the contract, $\rho=\mathrm{e}^{-\kappa}$, and the physical dynamics of the state variables in (3) are used to simplify the expression. In (6), $\Delta u_{t, T} \sim$ iid $\mathrm{N}\left(0,2 \sigma_{T}^{2}\right)$ since $\Delta u_{t, T}=u_{t, T}-u_{t-1, T}$.

Comparison of (6) and (4) reveals three shortcomings of the conventional approaches in modelling term structure of commodity prices. First, the factor loadings are determined by a small number of parameters defining the dynamics of the spot price series in the conventional term-structure models. Specifically, the factor loadings are exponentially decreasing in time-to-maturity for the ST factor, whereas they are unity for the LT factor. In contrast, factor loadings for both the LT and ST factors are specified by flexible, nonparametric functions in (4), which allows the extent that current market shocks are reflected into futures prices to exhibit very complex patterns and vary across contracts with different maturity dates. Second, while the variance of measurement error $u_{t, m}$ is allowed to vary across the contracts with different maturity date, it is assumed to be constant over the time-to-delivery, $s$, in conventional term-structure models. In contrast, the variance of idiosyncratic error in (4) is specified as a function of $s$ and this function is allowed to vary across contract delivery months. Third, the innovations to the state variables, $\omega_{i, m}(i=1,2)$, are assumed homoskedastic in the term structure models whereas their conditional variance is specified to follow a GARCH process in the POTS model. These restrictions imposed on the stochastic dynamics of the underlying factors, factor loadings, and variance of measurement errors can together result in biased estimates of not only underlying factors and measurement errors but also the parameters determining seasonal mean price and the market price of risk parameters in conventional term-structure models. These biases are expected to be of considerable size for commodities with significant storage costs and strong seasonality in demand/supply, which are to be examined empirically in the subsequent section.

\section{DATA AND ESTIMATION}

\subsection{Data and estimation methods}

In this study, I estimate three models. The first model is the two-factor model of Schwartz and Smith (2002). Here, I estimate the subset of the model parameters that appear on the model's first difference form (6). First differencing eliminates the deterministic variation in mean price level (as specified by $f(T)$ ), thus, it emphasizes the bias resulting from the restrictive specifications of factor loadings, temporal dynamics of factor variances, and variance of measurement errors. First differencing also makes the model directly comparable to the second model I estimate; the POTS model, as defined in (4). The third model is a composite model in which factor loadings are specified as in Schwartz-Smith 2-factor model (hence imposing restrictive specification on the stochastic dynamics of the latent factors), whereas the variance of measurement errors are specified by a non-parametric function as in the POTS model. Comparison of this model with the POTS model allows us to distinguish the bias in the estimated factor loadings and the bias in the estimated dynamics of the latent factors into two sources; the biases resulting from the misspecification of their own and those resulting from misspecifying the variance of measurement errors. 
I estimate the three models for futures prices of the following four commodities with different peculiarities:

- Crude oil: consumption goods with very weak seasonality in demand and supply,

- Natural gas: consumption goods with strong seasonality in demand,

- Corn: consumption goods with strong seasonality in supply, and

- Gold: investment goods with virtually no seasonality either in demand or supply.

Data examined in this study are daily settlement prices of futures contracts traded at the NYMEX (crude oil, natural gas, and gold) and CBOT (corn) for the period between 1984/1/1 and 2007/12/31 for corn and gold, 1984/4/1 and 2007/12/31 for crude oil, and 1991/4/1 and 2007/12/31 for natural gas. Because long-dated contracts do not trade actively, price of contracts for more than twelve months to maturity are excluded from the analysis. Excluding these observations leaves 70,800 prices among 307 contracts for crude oil, 52,780 prices among 223 contracts for natural gas, 43,831 prices among 168 contracts for gold, and 48,762 prices among 142 contracts for corn.

\subsection{Estimation Results}

Table 1 summarizes the results from the specification test. In short, the two factor model of Schwartz and Smith (2000) stipulating restrictive structures on the factor loadings is empirically not supported. Comparing between the POTS and composite model, the POTS model is generally preferred to the composite model for all commodities. Only when the SIC is used, the composite model is preferred to the POTS model for crude oil which exhibits little or no seasonality in demand and supply. What is surprising is that the POTS model is preferred to the composite model for gold for which the storage cost is not significant and no demand or supply seasonality is expected.

Figures 1 through 3 illustrate the factor loadings, variance of idiosyncratic error, and total variance of natural gas prices as implied by the estimates of the three models. The figures show these results only for natural gas, for which the difference across the three models in the estimated factor loadings and variance of idiosyncratic errors is most notable. ${ }^{3}$ In panel (a) of figure 1, the factor loadings in the estimated POTS model indicate two notable features. First, the estimated factor loadings increase as the contract approaches to the maturity date for all twelve contracts. Second, the estimated factor loadings in the last few months of trading are higher for the contracts maturing in winter than those in summer. The estimated factor loadings for the ST factor in panel (b) exhibit the same features but in greater magnitude than those observed for the LT factor. In addition, for all twelve contracts, the estimated factor loadings for the ST factor start increasing rapidly in May, before which they are virtually zero for all twelve contracts. This is because the natural gas price tends to be high in winter peak demand season and there is no inventory carried over from the end of winter to the spring when the price is the lowest. In other words, the inter-temporal price linkage breaks at the end of winter peak demand season. In panel (c), the variance of idiosyncratic error in the estimated POTS model exhibits strong seasonality as well as the time-to-maturity effect. Unlike the two common factors, the idiosyncratic errors are not contemporaneously correlated across concurrently traded contracts. This indicates that high volatility in last one month of trading, particularly for winter contracts, represents the markets shocks that are specific to each contact and are of very short-term nature.

Figure 2 indicates the factor loadings, variance of idiosyncratic error, and total variance of natural gas prices as implied by the estimated Schwartz-Smith two-factor model. The model imposes the LT and ST factors to follow Brownian Motion and MR process, respectively, which results in the factor loadings of the LT factor to be identical for all twelve contracts and constant over one year of trading horizon whereas those of the ST factor to decrease exponentially with the time-to-maturity of the contract at the identical rate for all twelve contracts. The model also imposes the variance of the measurement error to be constant over the trading horizon while it allows the variance to vary by contract delivery dates. The figure indicates that, for natural gas, the idiosyncratic error dominates in magnitude the two latent factors in determining the variance of the daily futures price changes. Even though the estimated factor loadings depict clear mean-reverting behaviour in panel (b), their magnitude even on the last day of trading is marginal relative to the variance of idiosyncratic error. Together with the low factor loadings of the LT factor (resulting from the low variance of the LT factor estimated in the model), the model implies that the daily price changes exhibit virtually no correlation across concurrently traded contracts with different maturity dates, which will imply virtually no opportunity for hedging price risks by taking spread positions.

Figure 3 indicates the results for the composite model. Unlike for the Schwartz-Smith two-factor model, the estimated variance of idiosyncratic error exhibits strong seasonality and time-to-maturity effects. The

\footnotetext{
${ }^{3}$ The results obtained for the other three commodities are not presented here but are available from the author upon request.
} 
estimated factor loadings exhibit the same pattern as for the Schwartz-Smith two factor model, yet they are much greater in magnitude for the composite model than for the Schwartz-Smith model. The estimated total variance indicates strong seasonality and time-to-maturity effects in patterns similar to those revealed by POTS model in figure 1. The results signify the importance of allowing flexible functional forms in specifying the variance of idiosyncratic error, even when a researcher estimates parameters in conventional term- structure models.

\section{CONCLUSION}

In this study, I compare a conventional two-factor term-structure model of commodity futures prices with an alternative approach of modeling the variance of daily futures returns directly by flexible, non-parametric functions. Empirical estimation of the models to four commodities; gold, crude oil, natural gas, and corn, reveal that the volatility of daily futures prices exhibits strong time-to-maturity effects as well as significant seasonal pattern in its levels and compositions (among two factors and idiosyncratic errors). These complex patterns in the volatility of commodity futures prices cannot be replicated by the conventional two-factor Gaussian model, due to the restrictive dynamics stipulated onto the underlying stochastic factors. The composite model performs reasonably well in capturing strong seasonality in the volatility of commodity prices. The result signifies the importance of specifying the variance of idiosyncratic error by flexible functional forms; allowing capture of both the time-to-maturity effect and the seasonality in patterns different among contracts with different maturity date. Misspecifying the variance of idiosyncratic error can result in very different parameter estimates in the conventional term-structure models, which cautions recent trend of allowing more flexible stochastic processes of latent factors while stipulating restrictive structures on the variance of idiosyncratic errors. Incorrect portrait of volatility dynamics as well as correlation among concurrently traded contracts with different maturity dates can lead the conventional term-structure models to suggest hedging strategies that are less effective than the strategy based on the model of futures returns.

\section{REFERENCES}

Hull, J.C. (2002). Options, Futures, and Other Derivatives. $5^{\text {th }}$ ed. Prentice Hall.

Lautier, D. (2005). Term structure models of commodity prices: A review. Journal of Alternative Investments, 8(1), 42-64

Schwartz, E.S. (1997). The Stochastic behavior of commodity prices: Implications for valuation and hedging. Journal of Finance, 52, 923-973.

Schwartz, E.S., and J.E. Smith. (2000). Short-term Variations and Long-term Dynamics in Commodity Prices. Management Science, 46, 893-911.

Smith, A. (2005). Partially overlapping time series: A new model for volatility dynamics for commodity futures. Journal of Applied Econometrics, 20, 405-422.

Suenaga, H., and A. Smith. (2011). Volatility Dynamics and Seasonality in Energy Prices: Implications for Crack-Spread Price Risk. Energy Journal, 32(3), 27-58.

Williams, J.C., and B.D. Wright. (1991). Storage and Commodity Markets. Cambridge University Press, New York.

\section{ACKNOWLEDGEMENT}

This research was conducted with the financial support from the ARC Discovery Grant (DP1094499).

\section{APPENDIX - TABLE AND FIGURES}

Table 1. Model Selection Test

\begin{tabular}{lllll}
\hline & CL & NG & GC & C \\
\hline AIC & & & & \\
POTS & $\mathbf{- 6 9 7 5 8 4}$ & $\mathbf{- 4 3 1 2 4 0}$ & $\mathbf{- 5 5 2 9 3 4}$ & $\mathbf{- 4 0 5 4 1 4}$ \\
Schwarts-Smith & -618573 & -393533 & -510150 & -400083 \\
Composite & -696753 & -423613 & -551778 & -402995 \\
\hline SIC & & & & \\
$\quad$ POTS & -694999 & $\mathbf{- 4 2 8 7 3 8}$ & $\mathbf{- 5 5 1 6 8 3}$ & $\mathbf{- 4 0 4 3 5 0}$ \\
Schwarts-Smith & -618427 & -393391 & -510064 & -400004 \\
Composite & $\mathbf{- 6 9 5 7 9 0}$ & -422681 & -551282 & -402564 \\
\hline
\end{tabular}


Suenaga, Misspecification in term structure models of commodity prices: Implications for hedging price risk

(a) Factor Loading 1

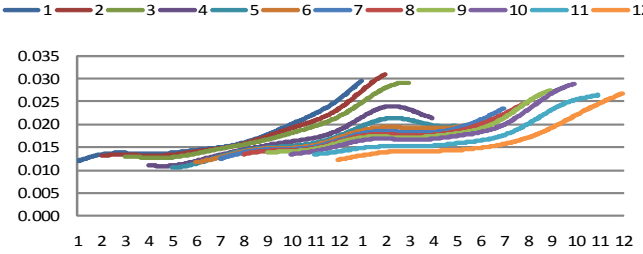

(b) Factor Loading 2

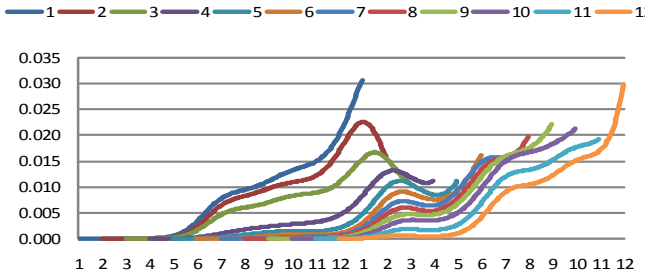

(c) Standard Deviation of Idiosyncratic Error

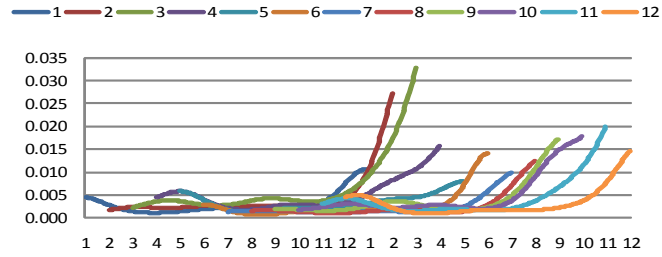

(d) Total Variance

Figure 1. Estimated factor loadings, variance of idiosyncratic error and total variance for POTS model

(a) Factor Loading 1

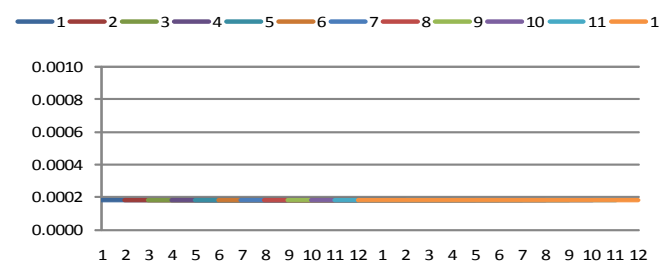

(b) Factor Loading 2

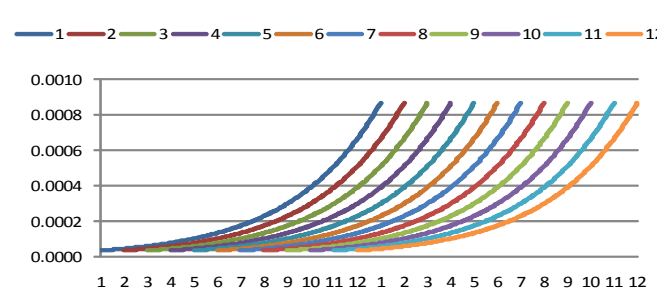

(c) Standard Deviation of Idiosyncratic Error

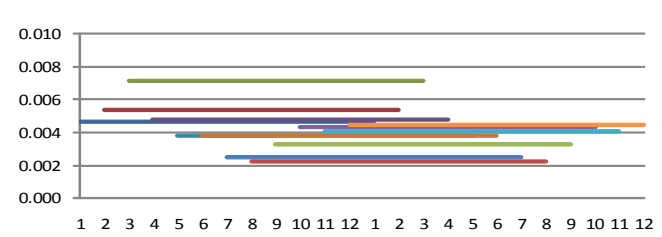

(d) Total Variance
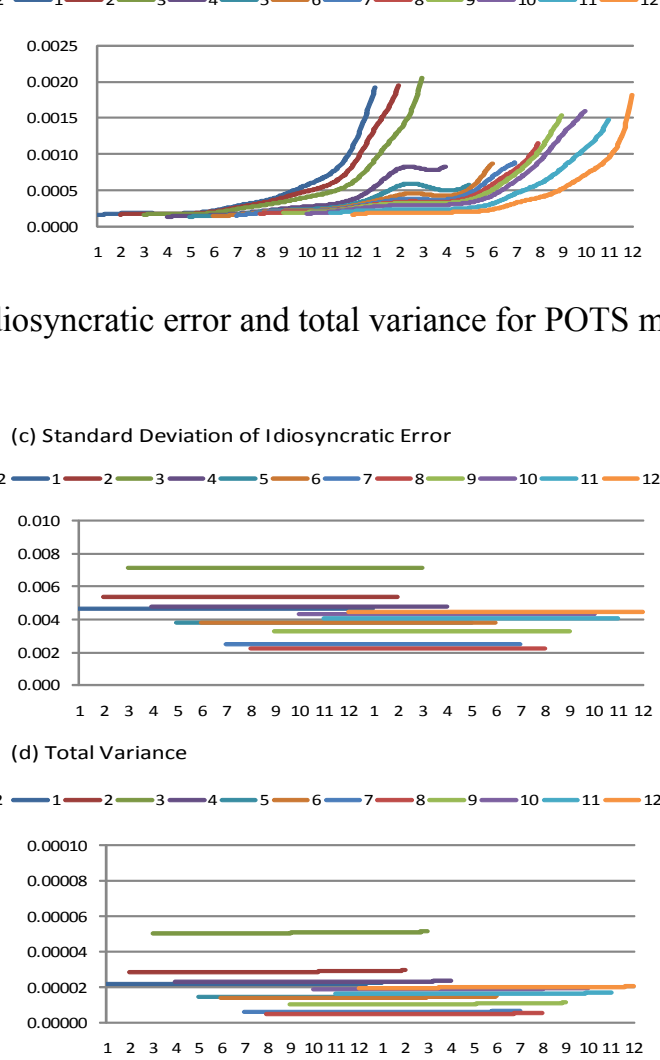

Figure 2. Estimated factor loadings, variance of idiosyncratic error and total variance for conventional termstructure model (Schwartz-Smith, two-factor model)

(a) Factor Loading 1

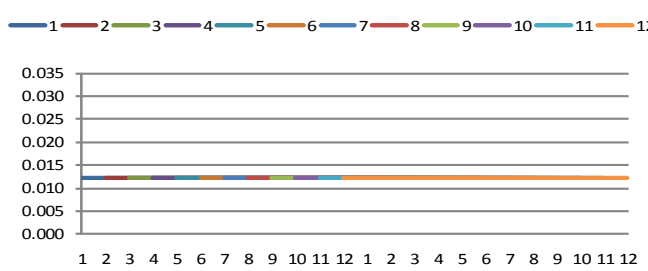

(b) Factor Loading 2

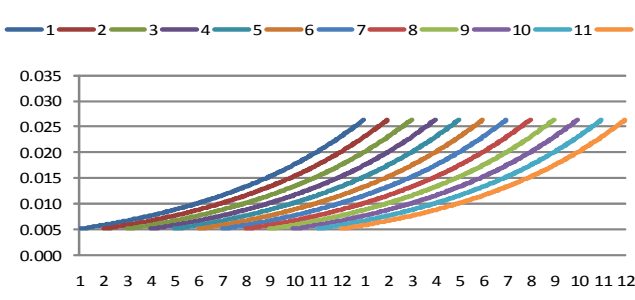

(c) Standard Deviation of I diosyncratic Error

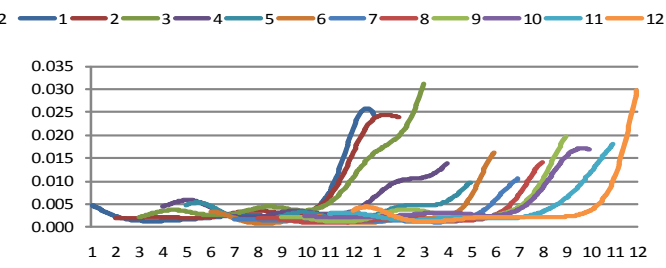

(d) Total Variance

Figure 3. Estimated factor loadings, variance of idiosyncratic error and total variance for composite model 